\title{
Hygiene and Wash Practices Associated with Urinary Tract Infection among Non-Pregnant Women at Capital Hospital, Bhubaneswar, Odisha, India
}

\author{
Jyoti Ranjan Mohanty ${ }^{1}$, Alisha Pradhan ${ }^{2}$, Sunita Jena ${ }^{2}$, P.R. Misra ${ }^{2}$, \\ Bijaya Padhi ${ }^{2}$ and Dhananjay Soren ${ }^{*}$ \\ ${ }^{1}$ Department of Zoology, Ravenshaw University, Cuttack, Odisha, India \\ ${ }^{2}$ Disease Surveillance Laboratory, Asian Institute of Public Health, \\ Bhubaneswar, Odisha, India \\ *Corresponding author
}

\begin{abstract}
A B S T R A C T

\begin{tabular}{|l|}
\hline Key w or d s \\
$\begin{array}{l}\text { Urinary tract infections, } \\
\text { Socio-economic status, } \\
\text { Uropathogens, Hygiene } \\
\text { practices }\end{array}$ \\
\hline Article Info \\
\hline $\begin{array}{l}\text { Accepted: } \\
\text { 16 February } 2018 \\
\text { Available Online: } \\
\text { 10 March } 2018\end{array}$ \\
\hline
\end{tabular}

Worldwide, urinary tract infection (UTIs) is one of the most common bacterial infections in women and it's depending on the individual's wash practices and socioeconomic status. The objectives of this study were to assess hygiene and wash practices and the associated factors of urinary tract infections in non-pregnant women, Odisha, in order to find this a cross-sectional study was carried out at Bhubaneswar, Capital Hospital from March 2015 to March 2017 in non-pregnant women of age group between 20-65 years. Out of 766 midstream urine samples (MSU), 323(42.1\%) samples were found to be UTI positive. From the defined age group, mainly 20-35 years age group women have showed the highest percentage $(64.09 \%)$ of infection. After adjusting the confounders, clinical recurrent infection (OR-3.65, 95\% CI 2.24-5.95, $\mathrm{p} \leq 0.001)$, vaginal wash (OR-0.10, 95\% CI $0.07-0.14, p \leq 0.001)$ and full body bath (OR- $0.26,95 \%$ CI $0.13-0.54 \mathrm{p} \geq 0.001)$. E. coli was the most predominant uropathogens among UTI infected non-pregnant women. Our findings indicate that there is an association between unhygienic practices and UTI and it is important that necessary hygiene practices should be used to prevent UTI.
\end{abstract}

\section{Introduction}

Urinary tract infections (UTI) are common bacterial infections found in the different age group of women (Gales et al., 1998). At least once in their lives, about half of the adult women reported urinary tract infections (Kunin, 1997). Globally, women have developed and followed their own personal stratagem to manage with their personal hygiene, which generally depends on financial condition, the individual's personal liking, 
Bangladesh study, conducted on both men and women concluded that UTI is greater in women $(48.29 \%)$ as compared to men $(38.44 \%)$, which may be either due to anatomical structure of the women. (Haque $e t$ al., 2015)

In community after respiratory tract infection, urinary tract infection (UTI) is most common type of infectious disease (Das et al., 2015). A study conducted in South part of India revealed that Women $(47.9 \%)$ had a significantly higher prevalence of UTI than men (Jennifer et al., 2009). Low socioeconomic status has also been found as risk factors for UTI (Peters et al., 2000). Some of the other risk factors are poor menstrual and toilet hygiene, use of tampons or douching and lack of awareness (Ness et al., 2005; Hillier et al., 1996).

A study conducted to determine the prevalence of community acquired-UTI among women in rural Odisha showed that prevalence of UTI in females was $45.2 \%$ (Das et. al., 2013). Urinary tract infections are believed to be among the most common form of infection in girls and women of menstruating age and this is held to be due to unhygienic practices (Groen et al., 2005). To the best of our knowledge, less data concerning hygiene practice which is associated with urinary tract infection among non-pregnant women from Odisha, India has been documented till date. We conducted the current study among non-pregnant women to assess the association between different hygiene practices and sociodemographic predictors with UTI.

\section{Materials and Methods}

\section{Study design and settings}

The cross-sectional study was conducted at Capital Hospital, Bhubaneswar, Odisha from
March 2015 to March 2017 Capital Hospital, a Government of Odisha hospital with 647 beds, has all major specialties including Obstetrics and Gynaecology $(\mathrm{O} \& \mathrm{G})$ and Family welfare.

\section{Study subjects}

Non Pregnant women of age group between 20-65 years who attended outpatient department (OPD) of $\mathrm{O} \& \mathrm{G}$ with symptoms of UTI were recruited in the study. Women with symptoms such as itching and burning while urinating, frequent urge to urinate, cloudy or blood in the urine were included. Diabetes, HIV infection and pregnant women who were menstruating at the time of assessment, had taken a course of antibiotics within last three weeks, and those who refused consent to participate were excluded from the study.

\section{Sample collection and processing of urine sample}

Mid-stream, clean catch urine sample was collected from symptomatic woman Urinary tract infections were diagnosed by culturing midstream urine sample in HiChrome UTI Agar (Himedia) plate. The plates were incubated in the presence of oxygen conditions at $37^{\circ} \mathrm{C}$ and read after 48 hours. Colony count $\geq 10^{5} \mathrm{CFU} / \mathrm{ml}$ and Plates having pure growth is considered as a standard for the experiment (Sumpter, et al., 2013). In case of less than $10^{5} \mathrm{CFU} / \mathrm{ml}$ colony count, the whole process with complete history was again repeated.

\section{Risk factor data collection}

A standardized tool was used and after collecting the urine sample, data were collected by trained female interviewers. The socio-demographic section contains all the characteristics including age; marital status, religion, caste, educational attainment, living 
arrangement, occupation, and wealth. Furthermore, wash practices such as only vaginal wash, a bath of full body and I don't wash types were also collected. Each woman was reassured that the all information's including biological samples will be kept confidential and used only for research purpose as well as the data-sheet were labeled with a unique identifier to ensure confidentiality of participants.

\section{Statistical analysis}

Pearson's chi-square test was used for comparison of the proportions and differences in values were considered significant if $P$ values were less than 0.05 . Multivariate analysis was used for socioeconomic, demographic and clinical factors to estimate the independent contribution of each variable.

The data were analyzed using the statistical program IBM Statistical package for social sciences (SPSS) 20

\section{Ethical approval}

The study was approved by the Institutional Review Board of Asian Institute of Public health (AIPH) (AIPH ethics ref: ERC/ No: 2015-09). Only those who provided written informed consent to participate in the study were included.

\section{Results and Discussion}

During the month of March 2015 to March 2017, a total number of 1067 non pregnant women between the age group of 20-65 years visited OG- OPD at the Capital Hospital, Bhubaneswar. Out of which, 179 were refused to give their consent and 122 were excluded from the study. Finally, 766 non Pregnant women were recruited and from this $323(42.16 \%)$ were found with urinary tract infection (Fig. 1).
UTI was found significantly higher in married women (1-15 years of marriage, 66.78\%) and in the age group between 20-35 years $(64.09 \%)$. Women belonging to OBC caste $\mathrm{t}$ $(53.40 \%)$, residing in urban slum area $(42.72 \%)$, as well as from village $(42.41 \%)$ and with lower monthly income of (INR5,000$10,000 /-) 43.34 \%$ were more prone to UTI with Other variables we couldn't find any significant difference between uninfected and infected UTI non, pregnant women (Table 1).

The most reported symptom was found in women was itching and burning while urination (69.97 \%), followed by vaginal discharge (45.20\%) and Genital sores (9.60\%).

However, recurrent infection was found to be more common among UTI infected women $(57.59 \%)$. No significant difference found in other variables such as Urinate frequently, Urine frequency, Cloudy blood in urine, Lower back pain, Stomach pain, Breast pain (Table 2).

Primary water source from the piped tap with $(64.09 \%)$ was found to be another variable for urinary tract infection followed by Wash practice $(71.52 \%)$. Other significant findings were Latrine/Bathroom Privacy (54.80\%) and Latrine Bathroom door (53.56\%) (Table 3).

Unadjusted univariate analysis of each of these associated factors is presented in the Univariate analysis showed women between the age group of 36-50 years with OR- (5.89), $95 \%$ CI 3.12-11.11, $\mathrm{p} \leq 0.001$ and in the age group between 20-35years with OR-(4.11), 95\% CI 2.26-7.48 $\mathrm{p} \leq 0.001$ were associated with UTI (Table 4).

Other risk factor such as Years of marriage between (16-30) with OR-(2.44), 95\% CI $1.15-5.20 \mathrm{p} \leq 0.020$ and (1-15) with OR-(2.83), 95\% CI 1.37-5.87, $\mathrm{p} \leq 0.005$ were considerably higher to urinary tract infection. 
Table.1 Socio-demographic characteristics of non pregnant women at capital hospital, Odisha $(\mathrm{N}=766)$

\begin{tabular}{|c|c|c|c|}
\hline & \multicolumn{2}{|c|}{ Urinary Tract Infection (UTI) } & \multirow[b]{2}{*}{ P-Value } \\
\hline Variables & $\begin{array}{l}\text { Without Urinary tract } \\
\text { infection }(\%)(\mathrm{N}=476)\end{array}$ & $\begin{array}{l}\text { With Urinary tract } \\
\text { infection }(\%)(\mathrm{N}=323)\end{array}$ & \\
\hline $\begin{array}{l}\text { Age } \\
1(20-35) \\
2(36-50) \\
3(51-65)\end{array}$ & $\begin{array}{l}273(61.63) \\
94(21.22) \\
76(17.16)\end{array}$ & $\begin{array}{l}207(64.09) \\
102(31.58) \\
14(4.33)\end{array}$ & 0.001 \\
\hline $\begin{array}{l}\text { Marital status } \\
\text { 1(Single, never married) } \\
\text { 2(Married) } \\
\text { 3(Widow) }\end{array}$ & $\begin{array}{l}41(9.26) \\
394(88.94) \\
8(1.81)\end{array}$ & $\begin{array}{l}28(8.67) \\
288(89.16) \\
7(2.17)\end{array}$ & 0.906 \\
\hline $\begin{array}{l}\text { Years of marriage } \\
1(1-15) \\
2(16-30) \\
3(31 \& \text { above })\end{array}$ & $\begin{array}{l}238(60.10) \\
123(31.06) \\
35(8.84)\end{array}$ & $\begin{array}{l}193(66.78) \\
86(29.76) \\
10(3.46)\end{array}$ & 0.013 \\
\hline $\begin{array}{l}\text { Caste/Tribe } \\
1 \text { (SC) } \\
2(\text { ST) } \\
3 \text { (OBC) } \\
4 \text { (Other caste) } \\
\end{array}$ & $\begin{array}{l}108(25.71) \\
8(1.90) \\
204(48.57) \\
100(23.81)\end{array}$ & $\begin{array}{l}69(22.33) \\
15(4.85) \\
165(53.40) \\
60(19.42)\end{array}$ & 0.043 \\
\hline $\begin{array}{l}\text { Education } \\
1 \text { (No formal education) } \\
2 \text { (Completed primary (5th year)) } \\
3 \text { (Completed secondary (6-10th year)) } \\
4(\text { Completed }+2 \& \text { above (university, } \\
\text { etc) }\end{array}$ & $\begin{array}{l}132(29.80) \\
172(38.83) \\
92(20.77) \\
47(10.61)\end{array}$ & $\begin{array}{l}116(35.91) \\
131(40.56) \\
53(16.41) \\
23(7.12)\end{array}$ & 0.083 \\
\hline $\begin{array}{l}\text { BPL card } \\
0 \text { (No) } \\
1 \text { (Yes) }\end{array}$ & $\begin{array}{l}260(58.69) \\
183(41.31)\end{array}$ & $\begin{array}{l}182(56.35) \\
141(43.65)\end{array}$ & 0.517 \\
\hline $\begin{array}{l}\text { Monthly Income } \\
1(\text { Bellow } 5,000) \\
2(5,000-10,000) \\
3(10,000-20,000) \\
4(20,000-30,000) \\
5(30,000 \text { and above }) \\
6(\text { Don't Know) } \\
\end{array}$ & $\begin{array}{l}62(14.00) \\
139(31.38) \\
125(28.22) \\
42(9.48) \\
8(1.81) \\
67(15.12)\end{array}$ & $\begin{array}{l}78(24.15) \\
140(43.34) \\
78(24.15) \\
16(4.95) \\
2(0.62) \\
9(2.79)\end{array}$ & 0.001 \\
\hline $\begin{array}{l}\text { Occupation } \\
\text { 1(Employed or self-employed) } \\
\text { 2(Housewife) } \\
\text { 3(Student) }\end{array}$ & $\begin{array}{l}89(20.09) \\
333(75.17) \\
21(4.74)\end{array}$ & $\begin{array}{l}66(20.43) \\
244(75.54) \\
13(4.02)\end{array}$ & 0.891 \\
\hline $\begin{array}{l}\text { Where live } \\
\text { 1(Housing colony) } \\
\text { 2(Urban slum) } \\
\text { 3(Rural/village) } \\
\text { 4(Hostel) }\end{array}$ & $\begin{array}{l}105(23.70) \\
155(34.99) \\
175(39.50) \\
8(1.81)\end{array}$ & $\begin{array}{l}32(9.91) \\
138(42.72) \\
137(42.41) \\
16(4.95)\end{array}$ & 0.001 \\
\hline $\begin{array}{l}\text { People live in house } \\
1(1 \& 2) \\
2(3-5) \\
3(5 \& \text { above })\end{array}$ & $\begin{array}{l}44(9.93) \\
280(63.21) \\
119(26.86)\end{array}$ & $\begin{array}{l}13(4.02) \\
212(65.63) \\
98(30.34)\end{array}$ & 0.008 \\
\hline
\end{tabular}




\section{Table.2 Clinical Illustrations among Non Pregnant Women at Capital Hospital, Odisha (N=766)}

\begin{tabular}{|c|c|c|c|}
\hline \multirow[b]{2}{*}{ Variables } & \multicolumn{2}{|c|}{ Urinary Tract Infection (UTI) } & \multirow[b]{2}{*}{ P-Value } \\
\hline & $\begin{array}{l}\text { Without Urinary } \\
\text { tract infection }(\%) \\
(\mathrm{N}=476)\end{array}$ & $\begin{array}{l}\text { With Urinary tract } \\
\text { infection }(\%) \\
(\mathrm{N}=323)\end{array}$ & \\
\hline $\begin{array}{l}\text { Urinate frequently } \\
0 \text { (No) } \\
1 \text { (Yes) } \\
\end{array}$ & $\begin{array}{l}318(71.78) \\
125(28.22)\end{array}$ & $\begin{array}{l}235(72.76) \\
88(27.24)\end{array}$ & 0.767 \\
\hline $\begin{array}{l}\text { Urine frequency } \\
\text { 1(Once) } \\
\text { 2(Two times a day) 3(Three or } \\
\text { more times) }\end{array}$ & $\begin{array}{l}23(18.40) \\
58(46.40) \\
44(35.20)\end{array}$ & $\begin{array}{l}8(9.09) \\
46(52.27) \\
34(38.64)\end{array}$ & 0.165 \\
\hline $\begin{array}{l}\text { Itching/burning when urinating } \\
0 \text { (No) } \\
1 \text { (Yes) }\end{array}$ & $\begin{array}{l}270(60.95) \\
173(39.05)\end{array}$ & $\begin{array}{l}97(30.03) \\
226(69.97)\end{array}$ & 0.001 \\
\hline $\begin{array}{l}\text { Cloudy/blood in urine } \\
1 \text { (Cloudy) } 2 \text { (Blood) } 3 \text { (Both } \\
\text { Cloudy \& Blood) } 4 \text { (No cloudy } \\
\text { and Blood) }\end{array}$ & $\begin{array}{l}125(28.22) \\
26(5.87) \\
45(10.16) \\
247(55.76)\end{array}$ & $\begin{array}{l}79(24.46) \\
16(4.95) \\
20(6.19) \\
208(64.40)\end{array}$ & 0.069 \\
\hline $\begin{array}{l}\text { Vaginal discharge } \\
0 \text { (No) } \\
1 \text { (Yes) } \\
\end{array}$ & $\begin{array}{l}202(45.60) \\
241(54.40)\end{array}$ & $\begin{array}{l}177(54.80) \\
146(45.20)\end{array}$ & 0.012 \\
\hline $\begin{array}{l}\text { Genital sores } \\
0(\mathrm{No}) \\
1 \text { (Yes) }\end{array}$ & $\begin{array}{l}371(83.75) \\
72(16.25)\end{array}$ & $\begin{array}{l}292(90.40) \\
31(9.60)\end{array}$ & 0.008 \\
\hline $\begin{array}{l}\text { Lower back pain } \\
0 \text { (No) } \\
1 \text { (Yes) }\end{array}$ & $\begin{array}{l}292(65.91) \\
151(34.09)\end{array}$ & $\begin{array}{l}220(68.11) \\
103(31.89)\end{array}$ & 0.524 \\
\hline $\begin{array}{l}\text { Stomach pain } \\
0 \text { (No) } \\
1 \text { (Yes) }\end{array}$ & $\begin{array}{l}317(71.56) \\
126(28.44)\end{array}$ & $\begin{array}{l}222(68.73) \\
101(31.27)\end{array}$ & 0.397 \\
\hline $\begin{array}{l}\text { Breast pain } \\
0 \text { (No) } \\
1 \text { (Yes) } \\
\end{array}$ & $\begin{array}{l}299(67.49) \\
144(32.51)\end{array}$ & $\begin{array}{l}210(65.02) \\
113(34.98)\end{array}$ & 0.473 \\
\hline $\begin{array}{l}\text { Clinic recurrent } \\
0 \text { (No) } \\
1 \text { (Yes) }\end{array}$ & $\begin{array}{l}251(56.66) \\
192(43.34)\end{array}$ & $\begin{array}{l}137(42.41) \\
186(57.59)\end{array}$ & 0.001 \\
\hline
\end{tabular}


Table.3 WASH practices among non pregnant women at capital hospital, Odisha $(\mathrm{N}=766)$

\begin{tabular}{|c|c|c|c|}
\hline & Urinary $T$ & Infection (UTI) & \\
\hline Variables & $\begin{array}{l}\text { Without Urinary tract } \\
\text { infection }(\%)(N=476)\end{array}$ & $\begin{array}{l}\text { With Urinary tract } \\
\text { infection }(\%)(\mathrm{N}=323)\end{array}$ & P-Value \\
\hline $\begin{array}{l}\text { Primary water source location } \\
1 \text { (In the house) } \\
2 \text { (In the yard) } \\
\text { 3(At a relative's/ neighbor's house or yard) } \\
4 \text { (At a public location) }\end{array}$ & $\begin{array}{l}277(62.53) \\
57(12.87) \\
13(2.93) \\
96(21.67)\end{array}$ & $\begin{array}{l}187(57.89) \\
50(15.48) \\
10(3.10) \\
76(23.53)\end{array}$ & 0.598 \\
\hline $\begin{array}{l}\text { Primary water source } \\
\text { 1(Piped tap) } \\
\text { 2(Tube well or borehole or protected well) } \\
\text { 3(Unprotected well) } \\
\text { 4(Tanker truck or cart with a small tank) } \\
\text { 5(Surface water (river, dam, lake, pond or } \\
\text { stream, canal, irrigation channel)) } \\
\end{array}$ & $\begin{array}{l}310(69.98) \\
85(19.19) \\
7(1.58) \\
41(9.26) \\
0\end{array}$ & $\begin{array}{l}207(64.09) \\
54(16.72) \\
9(2.79) \\
45(13.93) \\
8(2.48)\end{array}$ & 0.002 \\
\hline $\begin{array}{l}\text { Household latrine/bathroom } \\
0 \text { (No) } \\
1 \text { (Yes) } \\
\end{array}$ & $\begin{array}{l}200(45.15) \\
243(54.85)\end{array}$ & $\begin{array}{l}126(39.01) \\
197(60.99)\end{array}$ & 0.090 \\
\hline $\begin{array}{l}\text { Water for latrine } \\
\text { 1(Tap inside latrine) } \\
\text { 2(Bring it from my private tube well) } \\
\text { 3(Neighbour tube well } 1 \text { min from home) } \\
4 \text { (Public source within } 1 \text { min from home) } \\
5 \text { (Far source more than } 5 \text { min walk) } \\
\end{array}$ & $\begin{array}{l}250(56.43) \\
106(23.93) \\
22(4.97) \\
56(12.64) \\
9(2.03)\end{array}$ & $\begin{array}{l}186(57.59) \\
72(22.29) \\
12(3.72) \\
49(15.17) \\
4(1.24)\end{array}$ & 0.648 \\
\hline $\begin{array}{l}\text { Where urinate } \\
1 \text { (Facility in-house or yard) } \\
2 \text { (Facility in relative or Neighbors house or } \\
\text { yard) } \\
\text { 3(Facility in the community) } \\
4(\text { (Open space(bush/field/Pond)) } \\
\end{array}$ & $\begin{array}{l}349(78.78) \\
17(3.84) \\
\\
21(4.74) \\
56(12.64)\end{array}$ & $\begin{array}{l}241(74.61) \\
11(3.41) \\
20(6.19) \\
51(15.79)\end{array}$ & 0.461 \\
\hline $\begin{array}{l}\text { Wash practice } \\
1 \text { (Only vaginal wash) } \\
\text { 2(Bath of full body) } \\
3 \text { (I don't wash) }\end{array}$ & $\begin{array}{l}323(72.91) \\
22(4.97) \\
98(22.12)\end{array}$ & $\begin{array}{l}78(24.15) \\
14(4.33) \\
231(71.52)\end{array}$ & 0.001 \\
\hline $\begin{array}{l}\text { Latrine/bathroom privacy } \\
0 \text { (No) } \\
1 \text { (Yes) }\end{array}$ & $\begin{array}{l}152(34.31) \\
291(65.69)\end{array}$ & $\begin{array}{l}146(45.20) \\
177(54.80)\end{array}$ & 0.002 \\
\hline $\begin{array}{l}\text { Latrine/bathroom roof } \\
0 \text { (No) } \\
1 \text { (Yes) }\end{array}$ & $\begin{array}{l}130(29.35) \\
313(70.65)\end{array}$ & $\begin{array}{l}99(30.65) \\
224(69.35)\end{array}$ & 0.697 \\
\hline $\begin{array}{l}\text { Latrine/bathroom door } \\
0 \text { (No) } \\
1 \text { (Yes) }\end{array}$ & $\begin{array}{l}153(34.54) \\
290(65.46)\end{array}$ & $\begin{array}{l}150(46.44) \\
173(53.56)\end{array}$ & 0.001 \\
\hline $\begin{array}{l}\text { Latrine/bathroom lock } \\
0 \text { (No) } \\
1 \text { (Yes) }\end{array}$ & $\begin{array}{l}127(28.67) \\
316(71.33)\end{array}$ & $\begin{array}{l}106(32.82) \\
217(67.18)\end{array}$ & 0.218 \\
\hline $\begin{array}{l}\text { Hand wash facility } \\
0 \text { (No) } \\
1 \text { (Yes) }\end{array}$ & $\begin{array}{l}86(19.41) \\
357(80.59)\end{array}$ & $\begin{array}{l}73(22.60) \\
250(77.40)\end{array}$ & 0.283 \\
\hline
\end{tabular}


Table.4 Univariate and multivariate analysis with unadjusted \& adjusted or in different sociodemographic, clinical and wash practices $(\mathrm{N}=766)$

\begin{tabular}{|c|c|c|c|c|}
\hline Risk Factor & $\begin{array}{l}\text { Unadjusted } \\
\text { OR* (95\% CI*) }\end{array}$ & $P$ value & $\begin{array}{l}\text { Adjusted } \\
\text { OR }^{*}\left(95 \% \text { CI }^{*}\right)\end{array}$ & $P$ value \\
\hline $\begin{array}{l}\text { Age } \\
3(51-65) \\
2(36-50) \\
1(20-35) \\
\end{array}$ & $\begin{array}{l}1.0 \\
5.89(3.12-11.11) \\
4.11(2.26-7.48)\end{array}$ & $\begin{array}{l}0.001 \\
0.001\end{array}$ & $\begin{array}{l}1.0 \\
26.47(5.77-121.37) \\
18.90(3.48-102.53)\end{array}$ & $\begin{array}{l}0.001 \\
0.001\end{array}$ \\
\hline $\begin{array}{l}\text { Years of marriage } \\
3(31 \& \text { above }) \\
2(16-30) \\
1(1-15)\end{array}$ & $\begin{array}{l}1.0 \\
2.44(1.15-5.20) \\
2.83(1.37-5.87)\end{array}$ & $\begin{array}{l}0.020 \\
0.005\end{array}$ & $\begin{array}{l}1.0 \\
0.13(0.02-0.75) \\
0.21(0.03-1.40)\end{array}$ & $\begin{array}{l}0.022 \\
0.108\end{array}$ \\
\hline Caste/Tribe & $0.99(0.86-1.14)$ & 0.943 & $0.89(0.71-1.11)$ & 0.314 \\
\hline $\begin{array}{l}\text { Monthly Income } \\
5(30,000 \text { and above }) \\
4(20,000-30,000) \\
3(10,000-20,000) \\
2(5,000-10,000) \\
1(\text { Bellow } 5,000) \\
6(\text { Don't Know) } \\
\end{array}$ & $\begin{array}{l}1.0 \\
1.52(0.29-7.95) \\
2.49(0.51-12.05) \\
4.02(0.84-19.30) \\
5.03(1.03-24.55) \\
0.53(0.09-2.93)\end{array}$ & $\begin{array}{l}0.617 \\
0.255 \\
0.081 \\
0.046 \\
0.474\end{array}$ & $\begin{array}{l}1.0 \\
8.89(0.96-82.35) \\
5.12(0.58-45.23) \\
3.52(0.40-30.93) \\
4.21(0.43-40.63) \\
1.15(0.11-11.89)\end{array}$ & $\begin{array}{l}0.054 \\
0.141 \\
0.255 \\
0.213 \\
0.905\end{array}$ \\
\hline $\begin{array}{l}\text { Where live } \\
\text { 1(Housing colony) } \\
\text { 2(Urban slum) } \\
\text { 3(Rural/village) } \\
\text { 4(Hostel) }\end{array}$ & $\begin{array}{l}1.0 \\
2.92(1.84-4.61) \\
2.56(1.63-4.04) \\
6.56(2.57-16.74)\end{array}$ & $\begin{array}{l}0.001 \\
0.001 \\
0.001\end{array}$ & $\begin{array}{l}1.0 \\
1.82(0.86-3.82) \\
1.54(0.73-3.22) \\
2.84(0.61-13.13)\end{array}$ & $\begin{array}{l}0.113 \\
0.252 \\
0.181\end{array}$ \\
\hline $\begin{array}{l}\text { People live in house } \\
1(1 \& 2) \\
2(3-5) \\
3(5 \& \text { above }) \\
\end{array}$ & $\begin{array}{l}1.0 \\
2.56(1.34-4.87) \\
2.78(1.42-5.46)\end{array}$ & $\begin{array}{l}0.004 \\
0.003\end{array}$ & $\begin{array}{l}1.0 \\
1.42(0.54-3.71) \\
1.48(0.53-4.08)\end{array}$ & $\begin{array}{l}0.474 \\
0.448\end{array}$ \\
\hline $\begin{array}{l}\text { Itching/burning when urinating } \\
0 \\
1\end{array}$ & $\begin{array}{l}1.0 \\
3.63(2.68-4.93)\end{array}$ & 0.001 & $\begin{array}{l}1.0 \\
5.66(3.47-9.23)\end{array}$ & 0.001 \\
\hline $\begin{array}{l}\text { Vaginal discharge } \\
0 \\
1\end{array}$ & $\begin{array}{l}1.0 \\
0.69(0.51-0.92)\end{array}$ & 0.012 & $\begin{array}{l}1.0 \\
0.33(0.20-0.54)\end{array}$ & 0.001 \\
\hline $\begin{array}{l}\text { Genital sores } \\
0 \\
1 \\
\end{array}$ & $\begin{array}{l}1.0 \\
0.54(0.34-085)\end{array}$ & 0.008 & $\begin{array}{l}1.0 \\
0.34(0.16-0.72)\end{array}$ & 0.005 \\
\hline $\begin{array}{l}\text { Clinic recurrent } \\
0 \\
1\end{array}$ & $\begin{array}{l}1.0 \\
1.77(1.32-2.37)\end{array}$ & 0.001 & $\begin{array}{l}1.0 \\
3.65(2.24-5.95)\end{array}$ & 0.001 \\
\hline $\begin{array}{l}\text { Primary water source } \\
\text { 1(Piped tap) } \\
\text { 2(Tube well or borehole or protected well) } \\
\text { 3(Unprotected well) } \\
\text { 4(Tanker truck or cart with a small tank) } \\
\end{array}$ & $\begin{array}{l}1.0 \\
0.95(0.64-1.39) \\
1.92(0.70-5.25) \\
1.64(1.03-2.59)\end{array}$ & $\begin{array}{l}0.799 \\
0.201 \\
0.034\end{array}$ & $\begin{array}{l}1.0 \\
1.13(0.61-2.08) \\
2.27(0.55-9.40) \\
1.64(0.83-3.24)\end{array}$ & $\begin{array}{l}0.681 \\
0.254 \\
0.148\end{array}$ \\
\hline $\begin{array}{l}\text { Wash practice } \\
\text { 3(I don't wash) } \\
\text { 1(Only vaginal wash) } \\
\text { 2(Bath of full body) }\end{array}$ & $\begin{array}{l}1.0 \\
0.10(0.07-0.14) \\
0.26(0.13-0.54)\end{array}$ & $\begin{array}{l}0.001 \\
0.001\end{array}$ & $\begin{array}{l}1.0 \\
0.05(0.03-0.93) \\
0.22(0.08-0.61)\end{array}$ & $\begin{array}{l}0.001 \\
0.004\end{array}$ \\
\hline $\begin{array}{l}\text { Latrine/bathroom privacy } \\
0 \\
1\end{array}$ & $\begin{array}{l}1.0 \\
0.63(0.47-0.84)\end{array}$ & 0.002 & $\begin{array}{l}1.0 \\
0.93(0.57-1.51)\end{array}$ & 0.772 \\
\hline $\begin{array}{l}\text { Latrine/bathroom door } \\
0 \\
1\end{array}$ & $\begin{array}{l}1.0 \\
0.60(0.45-0.81)\end{array}$ & 0.001 & $\begin{array}{l}1.0 \\
0.40(0.24-0.68)\end{array}$ & 0.001 \\
\hline
\end{tabular}


Fig.1 Schematic diagram of patient recruitment

Number of Non Pregnant women between 20-65 years age group visited OG-OPD at Capital Hospital, Bhubaneswar during the period of study: 1067

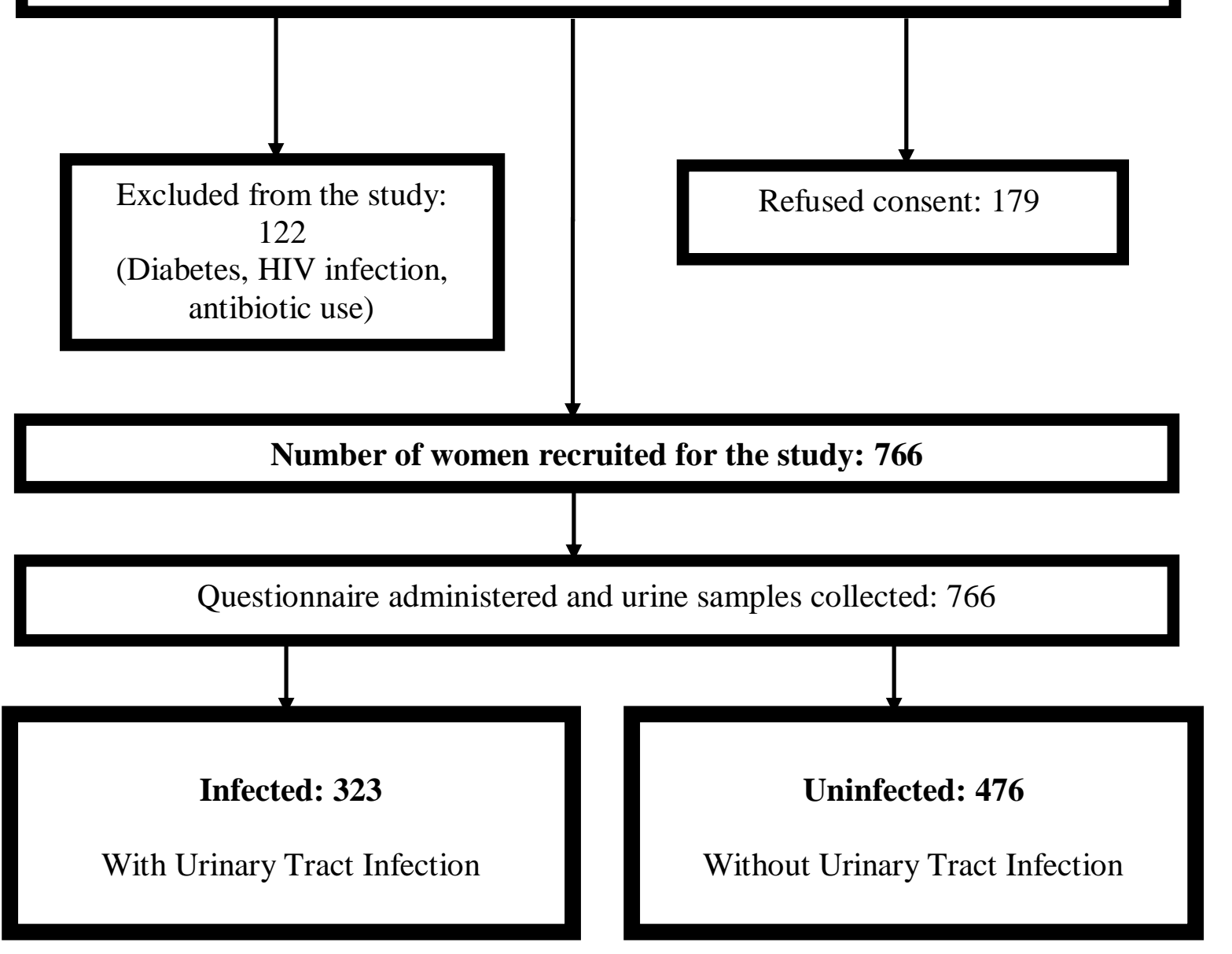

Risk of UTI (OR-2.92, 95\% CI 1.84-4.61, $\mathrm{p} \leq 0.001), \quad(\mathrm{OR}-2.56, \quad 95 \%$ CI 1.63-4.04, $\mathrm{p} \leq 0.001)$, (OR-6.56, 95\% CI 2.57- 16.74, $\mathrm{p} \leq 0.001)$ was also associated with the women who lived in Urban slum, Rural/Village \& Hostel respectively followed by people live in house between 3-5 with (OR-2.56, 95\% CI 1.34- 4.87, $\mathrm{p} \leq 0.004$ ) and $5 \&$ above (OR2.78, 95\% CI 1.42- 5.46, $\mathrm{p} \leq 0.003$ ). While Clinically Itching/ burning when urinating varies significantly (OR-3.63, 95\% CI 2.684.93, $\mathrm{p} \leq 0.001$ ) followed by Vaginal discharge (OR-0.69, 95\% CI 0.51- 0.92, $\mathrm{p} \leq 0.012$ ), Genital sores (OR-0.54, 95\% CI (0.34- 0.85), $\mathrm{p} \leq 0.008)$ and clinically recurrent (OR-1.77, 95\% CI 1.32- 2.37, $\mathrm{p} \leq 0.001$ ) were found to be associated with UTI in non-pregnant women.

Women assessed with Primary water source from tanker truck or cart with small tank showed significantly higher odds of UTI (OR1.64, 95\% CI 1.03- 2.59, $\mathrm{p} \leq 0.034)$. Other risk factors associated with wash practice such as vaginal wash (OR-0.10, 95\% CI 0.07- 0.14, $\mathrm{p} \leq 0.001$ ) and Bath of full body (OR-0.26, 95\% CI $0.13-0.54, \mathrm{p} \leq 0.001)$ was likely to have more UTI. Whereas, lack of bathroom privacy (OR-0.63, 95\% CI 0.47- 0.84, 
$\mathrm{p} \leq 0.002)$ and no door in latrine (OR-0.60, 95\% CI $0.45-0.81, \mathrm{p} \leq 0.001)$ showed strong association with UTI.

After multivariate analysis for each outcome women associated with UTI were found to be higher in age group (36-50) with (AdjOR26.47, 95\% CI 5.77- 121.37, $\mathrm{p} \leq 0.001)$ and age group between 20-35 years (AdjOR$18.90,95 \%$ CI $3.48-102.53, \quad \mathrm{p} \leq 0.001$ followed by Years of marriage (16-30) with (AdjOR- 0.13, 95\% CI 0.02-0.75, $\mathrm{p} \leq 0.022$ ), Clinically Itching/burning when urinating (AdjOR-5.66, 95\% CI 3.47- 9.23, $\mathrm{p} \leq 0.001$ ), Vaginal discharge (OR-0.33, 95\% CI 0.20 $0.54, \mathrm{p} \leq 0.001$ ), Genital sores (AdjOR-0.34, 95\% CI 0.16-0.72, $\mathrm{p} \leq 0.001)$, Clinic recurrent (AdjOR-3.65, 95\% CI 2.24-5.95, $\mathrm{p} \leq 0.001$ ), Wash Practice which includes Vaginal wash (AdjOR-18.90, 95\% CI 3.48- 102.53, $\mathrm{p} \leq 0.001$ ) and Bath of full body (AdjOR-0.22, 95\% CI 0.08- 0.61, $\mathrm{p} \leq 0.001)$. Similarly, in case of privacy with respect to latrine/ bathroom door (OR-18.90, 95\% CI 3.48$102.53, p \leq 0.001)$ showed significantly higher odds of UTI (Table 4).

Urinary tract infections (UTI) are major health curse that affects the majority of women in every year. This study provides support for the hypothesis that hygiene and wash practices can increase and lead to the risk of urogenital problems. UTI's among non-pregnant women is associated with poor hygienic conditions. However, other factors may also contribute to the problem as there are several causes of UTI infections. Bacteria are found to be the main cause of this infection and it affects a different part of the urinary tract which includes kidneys, ureters, bladder, and urethra. Particularly, bladder and urethra infections are mostly found in women (Manal et al., 2012). Our study sites also allowed us to represent diversified samples which include individuals from a different age, castes, class and socio-economic status with a range of wash practices and cleanliness. The main finding of this study are age group of non-pregnant women between 20-65years showed the highest percentage $(64.09 \%)$ and live in a $(42.72 \%), p \leq 0.001$. This has been observed in another crosssectional study that prevalence of bacteriuria in healthy women increases with age, in school among girls it was found to be $1 \%$ and in healthy community-dwelling women older than 80 years it was greater than $20 \%$ (Nicolle, 2003). Similarly, 43.34\% of women found to be more prone to UTI, those are having a lower monthly income of (Rs 5,000$10,000 /-)$. In our study, the statistically significant higher percentage was observed in Itching/ burning (69.97 \%) when urinating followed by vaginal discharge and Genital sores. However, in health outcomes, selfreported symptoms have been used in several cross-sectional studies, with the most common symptoms reported so far was abnormal vaginal discharge and itching in the genitalia (Sumpter et al., 2013). Our study revealed that about half of women $(57.59 \%)$ had recurrent episodes of UTI and this result agree with Hamdan et al., and Masinde et al., who mentioned that a history of previous UTI is an important risk factor for asymptomatic UTI. In case of hygiene and wash practices, our study showed that about $71.52 \%$ of women do not wash their genital organs properly and this leads to urinary tract infection. Whereas, this result agrees with Tchoudomirova et al., who mentioned that several hygiene practices such as washing genitals pre-coitus, washing genitals postcoitus, taking baths, frequent replacing of underwear and washing genitals from front to back were associated with a reduced frequency of UTIs (Tchoudomirova et al., 2002). Across these studies, these practices were found to be particularly sensitive in women and girls belonging to lower socioeconomic groups of rural areas (Das et al., 2015). 
The limitation is this is an observational study and so it's difficult to determine causality of the observed associations. Another limitation is that we only measured the association of hygiene practices related to urogenital diseases, but other infections could be associated, which is not included in this study.

Strengths of our work include a good sample size, use of a sophisticated microbiological laboratory to diagnose UTI and the fact that all of the interviewers involved were females, which assured a stress-free environment to discuss a stigmatized and sensitive topic.

It can be concluded that there is a strong association between hygiene practices and urinary tract infection. They were significantly exposed to recurrent infection and followed improper wash and sanitation practices.

It is important that women need to be educated about genital hygienic practices to avoid harmful uropathogens and reducing rate of vaginal infection. Training programs should be conducted which helps in counseling women about the genital hygienic practices. Further studies and more interventions were required and it must be explored to improve the health of women and avoid urinary tract infection. This study indicates that, in the context of women's health research, sanitation or hygiene is an important dimension.

\section{Declaration of Interests}

We have no conflict of interests.

\section{Acknowledgement}

We would like to express our sincere gratitude for the support from study participants, female interviewers and hospital nurses for assisting in this project.

\section{References}

Aniebue, U.U., et al., The impact of premenarcheal training on menstrualpractices and hygiene of Nigerian school girls,, Pan Afr Med J, Vol. 2, 2009, pp.9-12.

Baisley, K., et al., Bacterial vaginosisin female facility workers in north-western Tanzania: prevalence and risk factors. Sex Transm Infect, Vol.85, 2009, pp.370-375.

Dasgupta, A., et al., Menstrual Hygiene: How Hygienic is the Adolescent Girl? Indian J Community Med, Vol. 33, 2008, pp.77-80.

Das, P., et al., Menstrual Hygiene Practices, WASH Access and the Risk of Urogenital Infection in Women from Odisha, India. PLoS ONE, Vol. 10, 2015, pp 1-16.

Gales, A.C., et al., Activity and spectrum of 22 antimicrobial agents tested against urinary tract infection pathogens in hospitalized patients in Latin America: report from the second year of the SENTRY antimicrobial surveillance program (1998). J Antimicrob Chemother, Vol. 45, 2000, pp.295-303.

Groen, S., et al., The Course of Recurrent Urinary Tract Infections in NonPregnant Women of Childbering Age, the Consequences for Daily Life and the Ideas of the Patient. Nederlands Tijdschrift Voor Geneeskunde, Vol. 149, 2015, pp.1048-51.

Hillier, S.L., et al., Role of bacterial vaginosis-associated microorganisms in endometritis. American journal of obstetrics and gynecology, Vol. 175, 1996, pp.435-441.

Hinmann, F., The meaning of significant bacteriuria. JAMA, Vol. 184, 1963, pp. 727-28. 
Hooton, T.M., Recurrent urinary tract infections in women, Int $\mathbf{J}$ Antimicrob Agents, Vol.17, 2001,pp. 259-68.

Arul, K. C., et al., A cross sectional study on distribution of urinary tract infection and their antibiotic utilization pattern in Kerala, International Journal of Research in Pharmaceutical and Biomedical Sciences, vol. 3, 2012, pp. 1125-1130.

Kunin, C.M.1997. MD Urinary Tract Infections. In: Williams and Wilkins Baltimore, fifth ed.

Manal, F., et al., Moustafa and Entesar, M.M. Association between the Hygiene Practices for Genital Organs and Sexual Activity on Urinary Tract infection in Pregnant Women at women's health center, at Assiut University Hospital. Journal of American Science, Vol.8, 2012, pp. 515-522.

Ness, R.B., et al., Cluster analysis of bacterial vaginosis - associated microflora and pelvic inflammatory disease. American journal of epidemiology, Vol.162, 2005, pp. 585-590.

Nicolle, L.E., Asymtomatic bacteriuria; when to screen and when to treat. Infect Dis Clin North AM. Vol. 17, 2003, pp. 36794.

Peters, S.E., et al., Behaviors Associated with Neisseria gonorrhoeae and Chiamydia trachomatis: Cervical Infection among Young Womena Attending Adolescent Clinics. Clinical pediatrics, Vol. 39, 2000, pp. 173-177.

Seifu, Wubalem Desta and Alemayehu Desalegn Gebissa. 2018. "Prevalence and Antibiotic Susceptibility of Uropathogens from Cases of Urinary Tract Infections (UTI) in Shashemene Referral Hospital, Ethiopia." BMC Infectious Diseases 18(1):30. Retrieved (https://bmcinfectdis.biomedcentral.com /articles/10.1186/s 12879-017-2911-x).

Sumpter, C., et al., Systematic review of the health and social effects of menstrual hygiene management. PLoS One, Vol. 8, 2013.

Tchoudomirova, K., et al., History, clinical findings, sexual behavior and hygiene habits in women with and without recurrent episodes of urinary symptoms. Actaobstetricia et gynecologics Scandinavica, Vol. 77, 2002, pp 654656.

Seifu, W., et al., Prevalence and Antibiotic Susceptibility of Uropathogens from Cases of Urinary Tract Infections (UTI) in Shashemene Referral Hospital, Ethopia. BMC Infectious Diseases, Vol. 18, 2018, pp. 30: 34 .

\section{How to cite this article:}

Jyoti Ranjan Mohanty, Alisha Pradhan, Sunita Jena, P.R. Misra, Bijaya Padhi and Dhananjay Soren. 2018. Hygiene and Wash Practices Associated with Urinary Tract Infection among NonPregnant Women at Capital Hospital, Bhubaneswar, Odisha, India. Int.J.Curr.Microbiol.App.Sci. 7(03): 1743-1753. doi: https://doi.org/10.20546/ijcmas.2018.703.206 